\title{
Haematoloechus pyrenaicus n. sp. (Trematoda, Haematoloecbidae), parasite d'Amphibiens Anoures dans les Pyrénées
}

\author{
Par Claude COMBES
}

Trois espèces appartenant au genre Haematoloechus Looss sont actuellement connues en France :

H. variegatus (Rudolphi 1819) Looss 1899, rencontré chez Rana esculenta L. à Bordeaux (Bailenger et Chanseau, 1954), en Camargue (Rebecq, 1958) et à Richelieu (in Dollfus, 1961).

H. similis Looss 1899, rencontré chez Rana esculenta L. à Bordeaux (Bailenger et Chanseau, 1954).

Enfin, des métacercaires, recueillies par Viallot en 1870 (cf. Buttner, 1950), sont considérées par Odening (1960) comme appartenant à $H$. asper Looss 1899.

En 1963, nous avons recueilli dans les Pyrénées-Orientales plus de 80 exemplaires d'un Trématode, du genre Haematoloechus, ne semblant appartenir ni à l'une des espèces ci-dessus, ni à aucune autre espèce connue du genre Haematoloechus. Nous en donnons la description ci-après :

\section{HAEMATOLOECHUS PYRENAICUS n. sp.}

Hôtes: Rana temporaria temporaria L., Bufo bufo bufo L.

Habitat : poumons.

Localités: Rieutord, Puyvalador (canton de Mont-Louis, Pyrénées-Orientales).

\section{Matériel de description :}

2 individus récoltés en avril 1963 chez une $R$. temporaria mâle,

18 individus récoltés en septembre 1963, chez une $R$. temporaria mâle,

1 individu récolté en septembre $1963 \mathrm{chez}$ un Bufo bufo mâle,

60 individus récoltés en septembre $1963 \mathrm{chez}$ un Bufo bufo mâle. 
(Les dimensions et moyennes données dans la description qui suit concernent, pour les préparations in toto, une série de 45 individus).

\section{Description}

\section{CORPS :}

Sur le vivant, le corps est allongé, aplati dorso-ventralement, très déformable, surtout dans la région antérieure qui peut s'étirer jusqu'à devenir presque filiforme. Sur les préparations in toto (animaux fixés en état de légère compression), le corps présente un aspect en lancette caractéristique : les deux extrémités sont effilées régulièrement, l'extrémité postérieure étant à peine plus élargie que l'extrémité antérieure (fig. $1 a$ ).

Les dimensions du corps sont les suivantes (longueur et largeur mesurées sur des préparations in toto ; épaisseur sur coupes transversales et longitudinales sériées) :

Longueur: 7,6 à $13,7 \mathrm{~mm}$ (moyenne 10,1).

Largeur: 1,8 à $3,2 \mathrm{~mm}$ (moyenne $2,3 \mathrm{~mm}$ ).

Epaisseur : 0,5 à $0,9 \mathrm{~mm}$ (moyenne $0,7 \mathrm{~mm}$ ).

\section{Cuticule :}

Mince $(0,004$ à $0,005 \mathrm{~mm})$, lisse ; aucune spinulation n'a été constatée, ni sur le vivant, ni sur les préparations in toto, ni sur les coupes histologiques.

\section{Ventouses :}

Circulaires, non papilleuses.

Ventouse orale : sub-terminale ; diamètre : 0,39 à $0,67 \mathrm{~mm}$ (moyenne : $0,48 \mathrm{~mm}$ ).

Ventouse ventrale: un peu en arrière du tiers antérieur du corps; diamètre: 0,26 à $0,43 \mathrm{~mm}(0,32)$.

La distance entre les deux ventouses varie de 2,76 à $4,98 \mathrm{~mm}(3,41)$. Le rapport ventouse orale sur ventouse ventrale est égal à 1,50 en moyenne ; il varie peu autour de ce chiffre : le rapport le plus bas que nous ayons observé est égal à 1,36 , le plus haut à 1,72 . Sur le vivant, nous avons constaté que les parasites se fixent très faiblement à la paroi pulmonaire et se laissent extraire avec la plus grande facilité.

\section{TUBE DIGESTIF :}

Bouche: au centre de la ventouse antérieure. Prépharynx: absent. Pharynx : diamètre transversal : 0,18 à $0,24 \mathrm{~mm}(0,22)$; diamètre antéro-postérieur : 0,16 à $0,23 \mathrm{~mm}(0,20 \mathrm{~mm})$. Esophage : court $(0,25 \mathrm{~mm}$ environ), étroit $(0,07 \mathrm{~mm})$.

Caecums : s'étendent sur les côtés du corps, en suivant un tracé sinueux dû à la présence des autres organes (testicules, utérus) ; leur calibre est peu variable $(0,14$ à 
$0,20 \mathrm{~mm}$ ), sauf à leur extrémité où ils se dilatent légèrement. Ils se terminent à environ $1 \mathrm{~mm}$ de l'extrémité postérieure du corps, mais ils ont rarement la même longueur ; l'un des deux, soit le gauche, soit le droit, s'approche généralement davantage de l'extrémité du corps que son voisin. Ils sont toujours bourrés de globules sanguins en cours de digestion.

\section{APPAREIL ReProducteur MALE :}

Testicules: disposés en diagonale dans la région située en arrière de la ventouse ventrale; l'un des testicules est toujours nettement décalé en avant par rapport à l'autre ; c'est tantôt le gauche, tantôt le droit ; si l'ovaire est à gauche du corps, c'est le testicule droit qui est en avant ; si l'ovaire est à droite, c'est le testicule gauche. Cette variation ne nous paraît obéir à aucune statistique précise ; sur les 45 individus, nous en avons noté 26 avec le testicule droit en avant, les 19 autres montrant la position inverse.

Les deux testicules sont allongés dans le sens de l'axe de l'animal et leur longueur est toujours supérieure au double de leur largeur. Le testicule postérieur est toujours le plus développé.

Testicule antérieur: longueur : 1,80 à 4,17 mm (moyenne : 2,22 mm); largeur : 0,61 à $1,57 \mathrm{~mm}(0,93)$.

Testicule postérieur : longueur : 1,99 à $4,67 \mathrm{~mm}(2,68)$; largeur : 0,57 à $1,30 \mathrm{~mm}$ $(0,84)$.

Les testicules sont lobés, principalement sur leur bord externe (le bord interne est soit lisse, soit faiblement lobé). Le nombre des lobes varie de 5-6 à plus de 15 ; il dépasse 10 le plus souvent. La profondeur des entailles peut atteindre la moitié de la largeur du testicule ; plus généralement, elle est égale au tiers de cette largeur.

Canaux déférents: prennent naissance à l'extrémité antérieure des testicules, se dirigent vers l'avant et se jettent au même point, à l'extrémité postérieure de la vésicule séminale (fig. $1 \mathrm{~b}$ ).

Vésicule séminale: très longue, flexueuse, s'étend depuis le niveau de l'ovaire jusqu'au niveau du pharynx, où elle rejoint la partie postérieure de la poche du cirre ; son diamètre est régulier $(0,105 \mathrm{~mm}$ d'après les coupes sériées).

Poche du cirre : ventrale, mesure $0,440 \mathrm{~mm}$ de longueur sur $0,075 \mathrm{~mm}$ de diamètre ; elle débouche au pore génital commun qui est ventral, médian, situé exactement au niveau du bord antérieur du pharynx.

\section{APPAREIL. REPRODUCTEUR FEMELLE :}

Ovaire : situé juste en arrière de la ventouse ventrale, latéralement, soit dans la moitié droite, soit dans la moitié gauche du corps, ainsi qu'il a été dit plus haut. Sa longueur varie de 1,11 à $1,30 \mathrm{~mm}$ (moyenne : 1,18), sa largeur de 0,57 à $0,65 \mathrm{~mm}$ $(0,61)$. Il est profondément lobé sur son bord externe, presque régulier sur son bord interne; le nombre des lobes varie de deux à dix ; la profondeur des entailles dépasse la moitié de la largeur de l'organe. 
Oviducte : court et étroit; il prend naissance dans la région interne de l'ovaire. Canal de Laurer : absent.

Réceptacle séminal: très développé (jusqu'à $1,20 \mathrm{~mm}$ sur $0,92 \mathrm{~mm}$ ) ; il est au même niveau que l'ovaire, dans le côté opposé du corps, mais s'avance ventralement au-dessous de lui. Sur les coupes sériées, on observe, du côté dorsal, le canal du réceptacle séminal, très court $(0,020 \mathrm{~mm})$ et étroit $(0,003 \mathrm{~mm})$.

Glande de Mehlis : bien développée, elle est située au voisinage de l'ovaire, à peu près dans le plan de symétrie du corps.

Glandes vitellogènes : se présentent sous forme de groupes (ou rosettes) de follicules qui sont situés à droite et à gauche du corps, dorsalement, entre les limites suivantes : vers l'avant, ils s'approchent jusqu'à $1 \mathrm{~mm}$ environ de la ventouse buccale ; vers l'arrière, ils se terminent au niveau du milieu du testicule postérieur dans le côté du corps opposé à l'ovaire. Ces limites peuvent être légèrement dépassées, mais il n'existe jamais de vitellogènes dans l'espace post-testiculaire.

Le nombre de rosettes est typiquement de 20-22, le nombre de follicules par rosettes s'écarte peu de la douzaine.

Chaque rosette possède un canal vitellin ; les canaux d'un même côté du corps se réunissent pour former un vitelloducte latéral ; il existe deux vitelloductes transverses qui se jettent dans un vitelloducte impair, lequel débouche dans l'oviducte (fig. $1 \mathrm{~b}$ ).

Utérus : il occupe un volume très important; il possède une branche descendante et une branche ascendante qui forment de très nombreuses boucles, en particulier dans la région post-testiculaire ; il existe, d'une manière constante, des boucles utérines extra-caecales qui remontent depuis l'extrémité postérieure jusqu'au niveau du milieu du testicule postérieur; dans quelques cas, ces boucles extra-caecales atteignent le niveau du bord antérieur du testicule postérieur, mais elles ne le dépassent jamais vers l'avant.

Eufs : couleur brun clair à brun foncé. Forme régulière, moyennement allongée ; limite du clapet d'ouverture bien visible (fig. $1 \mathrm{c}$ ). Dimensions : 0,022 à $0,025 \mathrm{~mm}$ (moyenne : 0,023$)$ sur 0,014 à $0,017 \mathrm{~mm}(0,016)$.

\section{APPAREIL EXCRÉTEUR :}

La vessie n'est pas visible sur les individus montés in toto. Par contre, on l'observe très bien sur les coupes sériées. Elle forme un $\mathrm{Y}$ dont la branche médiane est beaucoup plus longue que les branches paires, la bifurcation se faisant au niveau du bord postérieur du réceptacle séminal. Par rapport à ce dernier, les branches paires sont en position latéro-ventrale ; vers l'avant, elles se terminent à $1 \mathrm{~mm}$ environ du pharynx.

\section{Epidémiologie}

Les deux $B$. bufo et les deux $R$. temporaria hébergeant $H$. pyrenaicus ont été capturés à très peu de distance (3 $\mathrm{km}$ environ) les uns des autres. Dans le même périmètre, nous avons examiné $32 \mathrm{~B}$. bufo et $21 \mathrm{R}$. temporaria; à proximité de cette station, dans les 

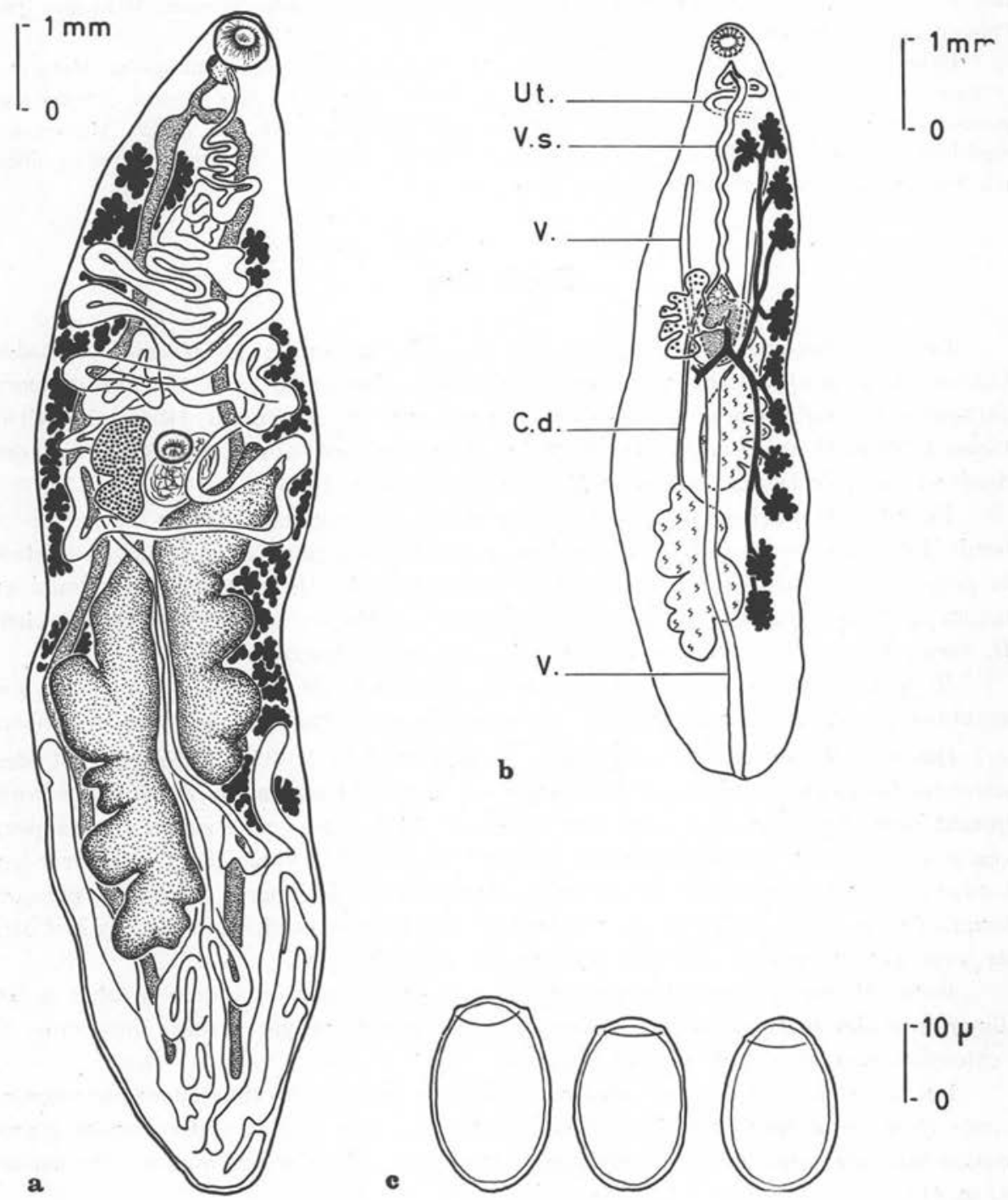

FIg. 1. - Haematoloechus pyrenaicus n. sp. : a) vue ventrale d'un individu in toto; $b$ ) dispoposition des organes génitaux ; $c$ ) cufs. C.d. $=$ canal déférent, Ut. $=$ utérus. V. $=$ vessie, V.s. = vésicule séminale

régions de la Cerdagne et du Capcir, nous avons disséqué $200 \mathrm{~B}$. bufo et $500 \mathrm{R}$. temporaria sans retrouver ce parasite; dans l'état actuel de nos observations, $H$. pyrenaicus est donc à la fois rare et extrêmement localisé. Son abondance par hôte est variable; chez l'un 
des $B$. bufo, nous avons noté que les poumons étaient considérablement déformés par l'abondance des parasites.

Dans la même région, nous n'avons rencontré aucune autre espèce du genre Haematoloechus, tandis que Haplometra cylindracea est assez abondant. Les quatre Amphibiens parasités par $H$. pyrenaicus n'hébergeaient aucun autre Trématode, ni aucun Monogène; signalons enfin que $B$. calamita, qui se rencontre en petit nombre dans des stations voisines, n'a été trouvé porteur d'aucun parasite pulmonaire.

\section{Discussion}

Les individus que nous décrivons font partie de la famille des Haematoloechidae Odening 1964 et appartiennent au genre Haemato'oechus Looss 1899. Certains de leurs caractères les rapprochent des espèces européennes $H$. variegatus (Rudolphi 1819) Looss 1899 et $H$. schulzei (Wundsch 1911), de l'espèce nord-américaine $H$. breviplexus Stafford 1905, de l'espèce africaine $H$. exoterorchis Rees 1964.

En effet, $H$. variegatus possède des testicules relativement allongés et parfois à bords irréguliers, et des œufs de dimensions comparables à celles de nos individus. Mais la présence de vitellogènes dans l'espace post-testiculaire, le caractère très limité et inconstant des entailles testiculaires, l'absence d'entailles ovariennes nettes chez $H$. variegatus sont en contradiction formelle avec notre description.

$H$. schulzei, malgré sa grande taille et les dimensions de ses œufs, s'éloigne de nos exemplaires par sa cuticule spinulée, ses testicules peu irréguliers, son ovaire ovoïde.

Quant à $H$. breviplexus, il possède, d'après Stafford (1905) et Cort (1915), des testicules fortement allongés et lobés, tantôt sur leur bord externe, tantôt sur leur bord interne, ainsi qu'un ovaire largement découpé. Mais, chez cette espèce, le rapport ventouse orale sur ventouse ventrale est égal à deux, les vitellogènes s'étendent en arrière du testicule postérieur et les lacets extra-caecaux de l'utérus remontent presque jusqu'au niveau de l'ovaire (la cuticule est spinulée d'après Stafford, lisse d'après Cort, de sorte que ce caractère ne peut être pris en considération).

Enfin, $H$. exoterorchis se rapproche de notre espèce par ses testicules lobés et les dimensions des œufs, mais s'en différencie principalement par l'ovaire piriforme et l'extension post-testiculaire des vitellogènes.

Les autres espèces du genre Haematoloechus présentent des différences plus importantes avec nos exemplaires. Nous considérons donc que ceux-ci appartiennent à une espèce nouvelle pour laquelle nous proposons le nom de Haematoloechus pyrenaicus n. sp. (1).

Les caractéristiques essentielles de cette espèce peuvent se résumér commẽ suit:

1) Corps de grande taille, rétréci aux deux extrémités, surtout à l'extrémité antérieure.

2) Testicules disposés en diagonale, de grande taille, très allongés, fortement entaillés (principalement sur leur bord externe).

(1) Nous remercions M. le Professeur Odening qui a bien voulu examiner nos préparations et nous confirmer que nos exemplaires appartiennent à une espèce nouvelle. 
3) Ovaire profondément entaillé.

4) Absence de vitellogènes dans la région post-testiculaire.

5) Lacets extracaecaux de l'utérus s'étendant jusqu'au niveau du milieu du testicule postérieur, ou un peu en avant de ce niveau.

Nous proposons ci-après une clé de détermination des espèces françaises du genre Haematoloechus :

1 Vitellogènes ne dépassant pas le niveau du testicule postérieur $\ldots \ldots \ldots \ldots \ldots \ldots 2$

1' Vitellogènes s'étendant dans la région post-testiculaire $\ldots \ldots \ldots \ldots \ldots \ldots \ldots \ldots$

2 Cuticule spinulée; ovaire et testicules peu irréguliers, non entaillés ..... H. similis

2' Cuticule lisse ; ovaire et testicules fortement entaillés ............ pyrenaicus

3 Cuticule spinulée; testicules elliptiques; œufs de 0,041 à $0,064 \times 0,020$ à

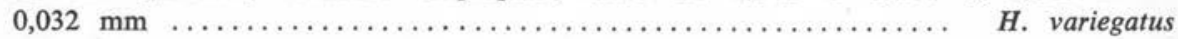

3' Cuticule lisse; testicules généralement allongés; œufs ne dépassant pas $0,033 \times$

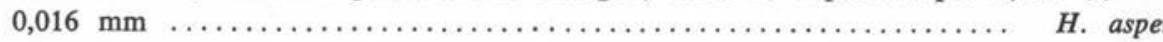

(Laboratoire de Zoologie et Biologie animale, Collège Scientifique Universitaire, Perpignan) (Pyr.-Orient.)

\section{Bibliographie}

Bailenger (J.) et Chanseau (J.), 1954. - Etude des vers parasites des Amphibiens Anoures de la région de Bordeaux. Ann. Par. Hum. Comp., XXIX, 5-6, p. 546-560, fig. 1-5.

Buttner (A.), 1950. - La progénèse chez les Trématodes Digénétiques. I. Ann. Par. Hum. Comp., XXV, 5-6, p. 376-434.

Cort (W. W.), 1915. - North American frog lung flukes. Trans. Amer. Micr. Soc., 34 (4), p. 203-239, pl. VII-IX.

Dollfus (R.-Ph.), 1961. - Station expérimentale de parasitologie de Richelieu (Indre-etLoire). Contribution à la faune parasitaire régionale. Ann. Par. Hum. Comp., 3, p. $169-451$, fig. 1-151.

OdenING (K.), 1958. - Zur Systematik von Haematoloechus (Trematoda, Plagiorchiidae). Mitt. Zool. Mus. Berlin, 34, 1, p. 63-108, fig. 1-13.

- 1960. - Trematoda, Digenea. Plagiorchiidae, III (Haematoloechinae) und Omphalometridae. Das Tierreich Lief. 75. Walter de Gruyter et Co., Berlin, p. 1-77, fig. 1-60.

- 1964. - Zur Taxionomie der Trematodenunterordnung Plagiorchata. Mon. Deutsch. Akad. Wiss. Berl., VI, 3, p. 191-198.

REes (G.), $1964 . \quad$ - Two new species of the genus Haematoloechus (Digenea: Plagiorchiidae) from Rana occipitalis (Gunther) in Ghana. Parasitology, 54, p. 345-368, fig. $1-29,1$ pl.

StAFFord (J.), 1902. - On the American representatives of Distomum variegatum. Zool. Jahrb. Syst., 16, p. 895-912, fig. 1-5. 